\title{
ELECTRONIC OPTICAL SKY SURVEYS
}

\author{
T.A. MCKAY \\ University of Michigan Department of Physics
}

\section{Introduction}

The introduction of of Charge Coupled Devices (CCDs) in the middle 1970s provided astronomy with nearly perfect (linear, high-sensitivity, low-noise, high dynamic-range, digital) optical detectors. Unfortunately, restrictions imposed by CCD production and cost has typically limited their use to observations of relatively small fields. Recently a combination of technical advances have made practical the application of CCDs to survey science. CCD mosaic cameras, which help overcome the size restrictions imposed by CCD manufacture, allow electronic access to a larger fraction of the available focal plane. Multi-fiber spectrographs, which couple the low-noise, high QE performance of CCDs with the ability to observe spectra for many objects at once, have improved the spectroscopic efficiency of telescopes by factors approaching half a million. An improved understanding of image distortion gives us telescopes on which we expect sub-arcsecond images a large fraction of the time. Finally, and perhaps most important, the performance of computer hardware continues to advance, to the point where analysis of multi-terabyte datasets, while still daunting, is at least conceivable.

Optical sky surveys occupy a special place in the astrophysical survey world. Due both to the information content of optical emission and to the great power of the global collection of optical instrumentation, no object is generally considered "understood" until it's optical counterpart is found. These electronic optical survey projects, providing accurate optical data for much of the sky, will thus have a major impact on surveys at all other wavelengths.

We discuss the status and prospects for several subclasses of surveys; time domain surveys, redshift surveys, and deep, wide-field imaging sur- 
veys. This is followed by a relatively detailed description of the Sloan Digital Sky Survey, which includes elements of all three. We conclude with some general considerations about the difficulties these surveys face, and what prospects they present for the future of astronomy. This discussion will concentrate on the instruments which enable surveys to take place. Many of these instruments will be used for a variety of survey projects, and it is their parameters which define what is possible.

\section{Time Domain Surveys}

The study of variable objects has taken an unsurpassed leap in the last ten years, driven by the ability of CCDs to provide accurate photometry of many objects at once. The achievements of the microlensing experiments represent the state of the art in these photometric monitoring programs. The scale of these experiments is set by the need to monitor millions of objects on a regular basis for several years. The MACHO project, for example, has been monitoring about 22 million stars in two colors for 4 years. These experiments have observed several hundred microlensing events, impressively vindicating the effort put into them. That said, the most remarkable new results of these projects are the variable star catalogs. The MACHO project alone has detected and heavily sampled at least 80,000 variable stars in every known class, and several unknown ones. This is to be compared with the 20-30,000 variable stars known across the entire sky in the early 90 's. The power of the increased sensitivity and accuracy of CCDs is demonstrated clearly here.

The new science being probed by the time domain surveys includes measures of the microlensing optical depth to the LMC (Alcock et al. 1996), a surprisingly high optical depth to the galactic bulge, the previously mentioned detailed studies of many classes of variable stars, new and challenging constraints on $H_{0}$ (Saha et al. 1996, Ferrarese et al. 1996), a deeper understanding of the details of Supernova light curves (Riess, Press, and Kirschner 1995), and the discovery of many type IA supernova at high redshift (Perlmutter et al. 1996). Prospects for the future in the time domain are directly connected to the construction of large area CCD mosaics intended for use on new telescopes.

\section{Redshift Surveys}

Redshift surveys are in many ways the progenitors of all electronic surveys. Starting from target lists selected as carefully as possible, they obtain homogeneous data sets designed for statistical study. The field of redshift surveys has been extremely active for the last 15 years (Strauss 1996, Colless 1996). Significant recent advances include the introduction and rapid 
advance of multi-fiber spectrographs, and the use of homogenous CCD photometry for target selection. Probably the premier wide area survey of the moment is the Las Campanas Redshift Survey, which has recently reported photometry and redshifts for about 26,000 galaxies in six 1.5 degree strips with a mean redshift of about 0.1 (Schectman et al. 1996). Also extraordinary are the narrow but deep redshift surveys like CFRS (Lilly 1996) and DEEP (Koo et al. 1996). In these surveys we're beginning to see samples of galaxies with median redshifts of 0.8 !

The progress rapid progress in wide area redshift surveys will be continued in the near future by two new projects; the 2DF (Taylor 1996) and the SDSS. The "Two-degree field" project will utilize a pair of robotically positioned 400 fiber spectrographs in combination with a focal reducer at prime focus of the AAT $4 \mathrm{~m}$. This instrument will be used to measure redshifts of 250,000 galaxies in a total of about 1700 square degrees. Galaxy targets will be selected from the APM scanned plates. Details of the SDSS are outlined below; essentially it will record spectra for $10^{6}$ galaxies over $10^{4}$ square degrees selected throughout from 5 color CCD data.

Redshift surveys are performed for two reasons; to study the intrinsic properties of galaxies, and to study the large-scale structure implied by their locations. Primary results on intrinsic properties include the galaxy luminosity function and the first really solid indications of galaxy evolution (Ellis et al. 1996, Lilly et al. 1996). Large-scale structure surveys continue to find unexpected structure at scales close comparable to the survey size, and have for several years been combined with independent distance indicators to study peculiar velocities and flows (Lauer and Postman 1994). As several large new telescopes come on line, studies of galaxy evolution will be done in which many galaxies at redshifts beyond one will be studied spectroscopically. Meanwhile the two new wide area surveys will expand the explored volume of the local universe by nearly a factor of 100 .

\section{Wide-Field Imaging Surveys}

CCD Imaging surveys of hundreds of square degrees of sky have only recently become practicable. They are enabled by the increased availability of large, easily mosaiced CCDs, along with the data acquisition and computing power required to handle the data. Perhaps the most exciting development in this area is the imminent availability of a number of new imaging detectors, each capable of performing deep imaging surveys on a photographic scale. The canonical new instrument has a field of view of several tens of arcminutes, instrumented with thinned CCDs, on a telescope of several meters aperture. The remarkable thing is that there are more than 10 of these instruments either online or planned for the near future. 
A good example of a camera already in operation is the BTC (Walker 1996). This camera places four thin CCDs at the prime focus of the CTIO $4 \mathrm{~m}$ to obtain a $30^{\prime}$ field of view. Spanning the range of cameras in development are a $21^{\prime}$ FOV prime focus imager for the SUBARU telescope and the 3 degree FOV SDSS imager. Data from these new cameras flows fast; a single image from the MMT $16 \mathrm{~K} \times 16 \mathrm{~K}$ camera will be half a gigabyte!

TABLE 1. New Wide-field Imaging Instruments

\begin{tabular}{lllll}
\hline Name & Pixels & FOV $^{\circ}$ & Telescope & Status \\
\hline BATC & $2 \mathrm{~K} \times 2 \mathrm{~K}$ Thin & 0.90 & $0.9 \mathrm{~m}$ BAO & Operational \\
QUEST & $4 \mathrm{~K} \times 4 \mathrm{~K}$ Thick & 1.3 & $1.0 \mathrm{~m}$ CIDA & In construction \\
LMT & $2 \mathrm{~K} \times 2 \mathrm{~K}$ Thick & 0.35 & $2.6 \mathrm{~m}$ LMT & Operational \\
BTC & $4 \mathrm{~K} \times 4 \mathrm{~K}$ Thin & 0.24 & $4.0 \mathrm{~m}$ CTIO & Operational \\
UH8K & $8 \mathrm{~K} \times 8 \mathrm{~K}$ Thick & 0.23 & $3.6 \mathrm{~m}$ CFHT & Operational \\
Kiso Camera & $5 \mathrm{~K} \times 8 \mathrm{~K}$ Thick & - & $4.2 \mathrm{~m}$ WHT & Operational \\
UH & $8 \mathrm{~K} \times 12 \mathrm{~K}$ Thin & 0.37 & $3.6 \mathrm{~m} \mathrm{CFHT}$ & In construction \\
UW $/$ APO & $8 \mathrm{~K} \times 8 \mathrm{~K}$ Thin & 0.13 & $3.5 \mathrm{~m} \mathrm{ARC}$ & In construction \\
SDSS imager & $\simeq 12 \mathrm{~K} \times 12 \mathrm{~K}$ Thin & 1.8 & $2.5 \mathrm{~m}$ SDSS & In construction \\
MMTCAM & $16 \mathrm{~K} \times 16 \mathrm{~K}$ Thin & 0.16 & $6.5 \mathrm{~m}$ MMT & In design \\
SubPrime & $10 \mathrm{~K} \times 8 \mathrm{~K}$ Thin & 0.14 & $8.3 \mathrm{~m}$ Subaru & In design \\
\hline
\end{tabular}

These instruments will be used for a broad range of science programs. Early deep CCD surveys discovered an unexpectedly large population of faint blue galaxies, which many of these instruments will use to measure weak gravitational lensing. Others will search for Kuiper belt and other faint nearby objects, or be used for the supernova search projects mentioned earlier. A special opportunity exists here; for many of these projects, particularly time domain surveys and deep co-added imaging, can utilize exactly the same data. It is hoped that collaborations can be designed to take advantage of the full range of science possible with deep survey data, so the time and effort required to produce them can be optimally used.

The particular advantages of CCDs have begun to make themselves felt, especially in the areas of time domain and redshift surveys. Having spent some time surveying the field, we will now concentrate on the SDSS, and use it to illustrate some of what is possible with new electronic surveys.

\section{The Sloan Digital Sky Survey}

The Sloan Digital Sky Survey will produce a digital image of the entire northern galactic cap ( $10^{4}$ sq.degrees). Data will be obtained with $0.4^{\prime \prime}$ 
resolution, in 5 colors, to 23rd magnitude in r'. From this imaging catalog, approximately $10^{6}$ galaxies and $10^{5}$ quasars will be selected. Spectra with a resolving power of about 2000 will be taken for all of these objects from about $400 \mathrm{~nm}$ to $910 \mathrm{~nm}$. In a complementary survey, a single $3 \times 100^{\circ}$ strip in the southern galactic cap will be imaged about 35 times over a five year period, providing both a large time domain survey and a deep co-added imaging survey to about 25 th magnitude. Imaging and spectroscopy will be done on the same telescope in an interleaved fashion; only photometric nights with sub-arcsecond seeing will be used for imaging. The SDSS (Gunn and Knapp 1993, see also http://www.astro.princeton.edu/GBOOK for details) is a collaboration of seven US and one Japanese institution, including over 200 scientists and engineers. Major support has been provided by the Alfred P. Sloan Foundation.

To conduct the survey we have constructed a new $2.5 \mathrm{~m}$ telescope at the Apache Point Observatory in southern New Mexico. The telescope features a novel modified Ritchy-Chretien design, which provides a flat, undistorted, 3 degree field of view, a focal plane about $27^{\prime \prime}$ in diameter. Median seeing at APO is $0.8^{\prime \prime}$; all the imaging will be done with sub-arcsecond seeing. The imaging camera for the survey, the largest CCD mosaic yet constructed, is being assembled at Princeton. There are six columns in the camera, each consisting of 5 SITe $2048 \times 2048$ pixel CCDs. Each of the five CCDs in a column views the sky through a different filter, allowing essentially simultaneous measurement in five colors. The camera will be operated in powered TDI mode, with 55 second effective exposures. A single pass through an area yields a "strip," a second interleaved pass fills the complete 2.6 degree wide "stripe." There are 54 CCDs with 145 million pixels in the imager, and it produces data at 8.2 Mbytes per second; 240 Gbytes in an 8 hour night.

The ultimate usefulness of the survey depends sensitively on the quality of the photometry. As the SDSS will observe most of the sky only once, we must carefully monitor all elements which affect photometry. An automated 24" "Monitor Telescope" has been constructed to measure atmospheric extinction and establish survey zeropoints on an hourly basis. We will also directly observe cloud cover with a $10 \mu \mathrm{m}$ imager. Careful astrometry is also needed, with limits set by our need to properly position the fibers for obtaining spectra. The required astrometric accuracy of 200 milliarcseconds should be immediately achievable, and recalibration with HIPPARCOS data may allow us to improve this to 50 milliarseconds.

In order to select targets and conduct spectroscopy within a month of obtaining imaging data, analysis of each 240 gigabyte night must be completed within one week. A custom data processing system has been developed which will perform the analysis. Much of this software and all 
of the hardware is in place, and we expect to be able to handle survey data essentially in real time by the time the survey begins.

Targets for SDSS spectroscopy will be selected from the SDSS imaging data. Galaxies will be chosen primarily by a magnitude cut; yielding a median redshift of about 0.1 . Quasars targets will be selected on the basis of their non-stellar colors; an area where accurate five color data is exquisitely useful. The spectra will be taken with a pair of dual channel spectrographs fed by 640 fibers. Ten manually plugged fiber cartridges will be prepared for each night; 6400 spectra on a good night. The spectrographs provide a resolution sufficient to measure typical galaxy velocities to $\pm 20 \mathrm{~km} \mathrm{sec}^{-1}$.

The SDSS promises to provide an unprecedentedly complete and detailed picture of the local universe. The spectroscopic survey will support large-scale structure studies in a fully sampled volume about 100 times that previously available, and will permit direct LSS studies with quasars for the first time. The combined imaging and spectroscopy will provide a measure of the galaxy luminosity function in five colors; luminosity/density and luminosity/morphology relations will be there to explore. The five color data will allow estimation of photometric redshifts for 50 million more galaxies with an estimated error of about $\delta Z=0.02$. The entire Northern Galactic Cap will have a 5 color calibration to a few percent; every field you look at will have objects accurately calibrated to 20th magnitude. And of course the most important discoveries will be those we haven't thought of.

All major telescope components are complete and the instruments are being delivered to the mountain this fall. We should begin taking data early next year, and begin the survey proper early in 1998. Data from the SDSS will be released to the public in as timely a fashion as is practical; data from the first two years will be released two years after they are taken, with the remainder of the survey released two years after it is completed. The "data" will consist first of complete object catalogs from all the imaging observations, a total of about 50 million galaxies, a million quasars, and 70 million stars and a spectroscopic catalog will including not only redshifts, but complete spectra. A second level of data release will consist of "atlas" images of every detected object. Finally the imaging observations will be collected into a single image of the full survey area.

\section{Conclusions}

Electronic surveys on a scale comparable to photographic surveys are now possible, and are being undertaken at a furious pace. This new generation of surveys is beginning to probe the universe in the spatial, time, and spectroscopic domains on a greater scale, and especially with greater accuracy, than ever before. Most of the technical impediments to conducting 
these surveys have been solved. We can construct large mosaics of highperformance CCDs, build spectrographs capable of measuring more than 500 spectra at once, and cajole our telescopes into providing sub-arcsecond images over large fields. A problem which all the projects will face, and which we have perhaps not yet satisfactorily solved, is the automated analysis and easy distribution of the immense quantity of data they electronic surveys will produce. Another point to note is that the most powerful and successful of these surveys all utilize dedicated equipment designed specifically for the purpose. Serious surveys require a serious commitment, and cannot generally be conducted as side projects.

Major discoveries made by electronic surveys so far include the faint blue galaxy population, many examples of weak and strong gravitational lensing and microlensing, the first real measures of large-scale structure and flows, and a more detailed quantitative understanding of SNe.

Just as the Palomar survey provided targets for $4 \mathrm{~m}$ class telescopes for 40 years, surveys like the SDSS will provide the basic survey material required for the selection of targets by the new generation of $8-10 \mathrm{~m}$ telescopes coming on line now. They will also provide the optical counterpart identification for many of non-optical surveys reviewed in these proceedings. These surveys, combined with the ready accessability of the immense quantities of data they will produce, hold the promise of again revolutionizing our view of our environs. It is an exciting time, a step forward in survey science which will probably not occur again for a generation.

\section{References}

Alcock, C., et al. 1996 Astrophys.J. Submitted

Colless, M., 1996 Proc. of the Athens conf. on 'Wide Field Spectroscopy,' in press

Ellis, R., et al. 1996 Mon.Not.R.astron.Soc. 280, 235

Ferrarese, L., et al. 1996 Astrophys.J. 464, 568

Gunn, J., and Knapp, G., 1993 in "Sky Surveys," ASP Conf. 43, 267

Koo, D., et al. 1996 Astrophys.J. Accepted

Lauer, T., and Postman, M., 1994 Astrophys.J. 425, 418L

Lilly, S., et al. 1996 Astrophys.J. 460, 1L

Perlmutter, S., et al. 1996 Astrophys.J. Submitted.

Riess, A., Press, W., and Kirschner, R., 1995, Bull.Am.Astron.Soc. 187, 1712

Saha, A., et al. 1996 Astrophys.J. 466, 55

Schectman, S., et al. 1996 Astrophys.J. 470, 172.

Strauss, M., 1996 Proceedings of Jeruselum Winter School, in press

Taylor, K., 1996 this volume, 135

Walker, A., 1996 this volume, 129 